\title{
Obstetric and Perinatal
}

\section{Complications Associated with}

Assisted Reproductive Techniques -

Review

\author{
Andreea Madalina BANICA ${ }^{a, b}$, Simona Daniela POPESCU ${ }^{a, b}$, Simona VLADAREANU ${ }^{a, b}$ \\ a"Carol Davila" University of Medicine and Pharmacy, Bucharest, Romania \\ bDepartment of Neonatology, "Elias" University Emergency Hospital, Bucharest, Romania
}

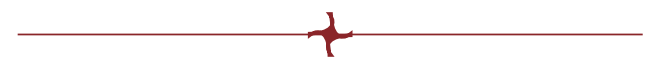

CABSTRACT

Recently, the use of assisted reproductive techniques (ART) has witnessed a significant increase worldwide. Although most of these pregnancies have a good prognosis, studies show that ART is associated with a risk of obstetric and perinatal complications, compared to pregnancies conceived spontaneously. It is considered that the risk is directly proportional to the number of transferred embryos, thus multiple pregnancies are an independent risk factor that supports the large-scale implementation of single embryo transfer protocols. Simultaneously, studies report obstetric and perinatal complications in singleton pregnancies obtained after $A R T$ and it is not possible to establish whether parental or procedural factors are the causal determinants. The purpose of this article is to summarize the risk of maternal-fetal complications associated with ART.
\end{abstract}

Keywords: assisted reproduction technology (ART), obstetric complications, neonatal complications, elective single embryo transfer (eSET), cryopreservation.

\section{INTRODUCTION}

nfertility is a public health problem with major implications for the physical, mental and social condition of affected couples. For this reason, most patients diagnosed with infertility decide upon using assisted reproductive techniques (ARTs) and studies are demonstrating an increase in their efficiency and safety over time (1). In 2016, in Europe, 1.8 million children were conceived through assisted reproduction (2).

Studies have shown that the use of ART was associated with an increased risk of obstetric complications such as pregnancy-induced hypertensive disorders, placental abnormalities, gestational diabetes, but also perinatal complications such as prematurity, low birth weight, small for gestational age and congenital anomalies. These side effects have been attributed to the increased incidence of multiple pregnancies, especially in older women (3). However, studies have shown that the risk of complications was maintained in the case of singleton pregnancies obtained after ART compared to the general population (4).

Recently, ART pregnancies have a better prognosis compared to previous years due to imple-

\footnotetext{
Address for correspondence:

Andreea Madalina Banica

"Elias" University Emergency Hospital, 17 Blvd. Mărăști, Bucharest, Romania

Tel.: 0740178414

Email: aandreeabanica@gmail.com
}

Article received on the $7^{\text {th }}$ of June 2021 and accepted for publication on the $3^{\text {rd }}$ of September 2021 
mentation of single embryo transfer protocols, which have reduced the incidence of multiple pregnancies and their associated complications. In Europe, the transfer of a single embryo increased from $37.7 \%$ in 2015 to $41.5 \%$ in 2016, and the transfer of two embryos decreased from $53.9 \%$ to $51.9 \%$. In 2016, two more countries, Slovenia and the United Kingdom, joined the single embryo transfer protocol, so that 10 European countries decided on this protocol (2).

Protocols using cryopreservation techniques increased the rate of single embryo transfer, which helped reduce the incidence of multiple pregnancies. According to statistics, cryopreservation techniques are beginning to gain significant importance, thus the transfer of cryopreserved embryos is increasing at European level (2). The implementation of the freeze-all protocol is currently taken into consideration because a reduction in the rate of multiple pregnancies and ovarian hyperstimulation syndrome has been observed. However, women should be carefully selected considering obstetric and perinatal risks associated with cryopreservation (5).

A study from the CoNARTaS group (6) demonstrated a decreasing trend of ART-associated complications compared to spontaneously obtained pregnancies. The increasing rate of single embryo transfer, use of cryopreservation techniques, as well as the shortening of the infertility period by early use of assisted reproduction can explain the improved prognosis in these pregnancies.

The current review provides a summary of data from the literature on maternal-fetal complications in pregnancies obtained through ART.

\section{Obstetric complications}

The results of studies in the literature show that pregnancies conceived through ART have an increased risk of obstetric complications such as pregnancy-induced hypertension, pre-eclampsia, placental abnormalities and gestational diabetes.

Pregnancy-induced hypertension and preeclampsia are common complications among women who get pregnant through ART (7-9). It has been observed that the risk of these complications is maintained also with cryopreservation $(5,10,11)$ and in the case of using donated fresh oocytes (12-15). Although some studies support the widespread use of cryopreserved embryos, the results are not conclusive.

The most recent meta-analysis comparing the use of freeze-all strategy with the transfer of fresh embryos did not report significant differences in the cumulative rate of live births. However, it was observed that the transfer of cryopreserved embryos increased the risk of hypertensive complications in pregnancy (16). Researchers assessed whether this effect was influenced by women's response to the ovarian stimulation protocol. Thus, two meta-analyses showed that, although the elective transfer of cryopreserved embryos was associated with an increased cumulative live birth rate only for the hyperresponsive population, it increased the risk of pre-eclampsia. This is the reason why women who can benefit from this procedure should be carefully selected $(17,18)$. The transfer of cryopreserved embryos is performed in the context of programmed cycles, in which estrogen and progesterone are administered to prepare the endometrium. In these cycles, ovulation is suppressed, so the luteal body is absent. Corpus luteum also secretes vasoactive hormones in addition to estrogen and progesterone. The role of these vasoactive hormones is to influence the development of the placenta, but they are not exogenously administered in programmed cycles (19). Because arterial compliance increases physiologically in pregnancy, von Versen-Höynck et al evaluated the velocity of the carotid-femoral pulse wave depending on the presence or absence of the luteal body. The results show that the absence of the luteal body decreases aortic compliance and increases the risk of pre-eclampsia in pregnancies obtained by in vitro fertilization (IVF) (20). This encourages the use of natural cycles when using cryopreservation techniques.

Beside that advanced maternal age and multiple pregnancies are independent risk factors for gestational diabetes (GD), ART use increases this risk by $28 \%$ (21). A meta-analysis compared singleton pregnancies obtained through ART with those obtained spontaneously and showed that assisted reproduction was associated with an increased risk of GD. This risk is higher in the IVF group compared to the intracytoplasmic sperm injection (ICSI) group and for fresh embryo transfer, compared to cryopreserved embryo-transfer (22). In a meta-analysis of 29 studies, Sha et al 
demonstrated that the risk of GD is higher in women diagnosed with polycystic ovary syndrome who obtain a pregnancy through IVF (23).

The risk of placental abnormalities, such as placenta previa and placental abruption, is increased in singleton pregnancies obtained through ART. A meta-analysis performed by Vermey et al demonstrates that this risk is also maintained in the population of subfertile women (24). Given the increased risk of pregnancy complications associated with multiple pregnancies, Karami et al performed a meta-analysis and demonstrated the association between ART use and the risk of placenta previa for both singleton and multiple pregnancies (25). The stage of embryo-transfer can also influence the risk of placental abnormalities. It is considered that the transfer of a blastocyst stage embryo increases the incidence of placenta previa (26). This can explain why the incidence of prematurity is higher in pregnancies obtained after transferring a day 5-embryo (27). Spangmose et al also demonstrated that the placenta previa rate was reduced if cryopreservation techniques were used compared to the transfer of fresh embryos. This result can be due to reduced endometrial stimulation in the case of cryopreservation (26).

\section{Perinatal complications}

A recent review by Madrazo-Cabo et al showed that pregnancies obtained through ART had an increased risk of preterm birth, low birth weight, small for gestational age, admission in neonatal intensive care unit (NICU) and congenital anomalies compared to the general population (28). A meta-analysis from 2013, which included 65 articles and analyzed the risk of prematurity, low birth weight and perinatal mortality, showed that the incidence of perinatal complications was lower in the ICSI group compared to the IVF group (29). Pregnant women in the ICSI group are considered reproductively healthier, as the primary indication for ICSI use is male infertility. Other studies claim that there are no significant differences between the two techniques in terms of the risk of complications (4). Cavoretto et al demonstrated in a meta-analysis that pregnancies obtained through IVF/ICSI had an increased incidence of spontaneous preterm birth compared to those obtained by spontaneous con- ception (10.1\% versus $5.5 \%)$, even after adjustment for potential confounders (30).

The use of blastocysts is considered to allow high-quality self-selection of embryos and thus increase the success rate of pregnancy and the rate of live births (31). However, Levi-Setti et al did not demonstrate any significant differences between the two groups (32). Simultaneously, Dar et al have shown that blastocyst embryo transfer was associated with an augmented risk of prematurity and low birth weight (27). To prevent the increased rate of prematurity and its complications in ART pregnancies, researchers analysed whether this risk was influenced by cryopreservation techniques. Maheshwari et al performed a meta-analysis showing that the transfer of a cryopreserved embryo was associated with a reduced risk of prematurity, compared to pregnancies obtained after transferring fresh embryos (10).

It has been demonstrated that both parental factors and assisted reproductive techniques could increase the risk of neonatal complications. However, the subfertile population of women remains uncontrolled in some studies as they are frequently included in the cohort of pregnancies obtained by spontaneous conception. In this case, sibling-control studies are needed to demonstrate the contribution of parental and obstetrical factors, regardless of the use of ART (33). Thereby, Romundstad et al analyzed the group of "sibling pairs" women who obtained a pregnancy both spontaneously and through ART. The results showed a similar rate of prematurity, suggesting that maternal factors and the etiology of infertility can influence this risk (34).

Among the perinatal complications, it has been shown that newborns obtained by ART have an increased risk of low birth weight and macrosomia compared with spontaneous births. Low birth weights are three times more commonly associated with ART. Multiple pregnancies and prematurity are independent risk factors, but the culture medium used and the ovarian stimulation protocol can also be responsible for the weight differences in these newborns (35). Castillo et al observed in a multicenter cohort study a trend of increasing birth weight of newborns from pregnancies obtained through IVF. In their study, the authors observed an increase in birth weight by 13.4 grams per 
year during the study period (36). Simultaneously, it was observed that newborns obtained after ART were at risk of large for gestational age and macrosomia, especially if cryopreservation techniques were used $(10,11,37)$. By transferring cryopreserved embryos, the selection of high-quality embryos is made and these pregnancies are considered to have a better prognosis because they do not require ovarian stimulation. However, adverse reactions associated with cryopreservation, such as pregnancy-induced hypertensive disorders, large for gestational age and perinatal mortality, have also been described in the literature. For this reason, the implementation of "freeze-all" protocols should be performed after carefully selecting the population of women who can benefit from this technique (38).

A meta-analysis from 2018 regarding congenital anomalies showed an increased risk in the ART pregnancy group with significant differences globally, depending on the procedure used and the time of diagnosis (39).

Congenital anomalies have been reported for the central nervous system, auditory and visual as well as cardiovascular, gastrointestinal and genitourinary systems. Even if these anomalies are frequently associated with multiple pregnancies obtained after ART (40), some studies show opposite results (41). Giorgione et al demonstrated that congenital heart defects were common in pregnancies obtained through ART (1.30\%) compared to those obtained spontaneously $(0.68 \%)$ for both singleton and multiple pregnancies (42).

Given that ICSI is more frequently associated with congenital anomalies than IVF (43), its use beside the indication of male infertility should be limited. That is the reason why it is recommended to properly select couples who can benefit from the use of ICSI.

Studies have shown that azoospermia or oligozoospermia was associated with an increased incidence of de novo chromosomal abnormalities compared with the normozoospermia group. An increased incidence of autosomal translocations and Y-chromosome microdeletions was observed too $(33,44)$.

Also, ART has been associated with the risk of diseases caused by gene hypomethylation such as Angelman syndrome, Beckwith-Weidermann syndrome, Prader Willi syndrome and Silver Russell syndrome (45). However, given the rarity of these pathologies, most studies do not have sufficient statistical power to demonstrate a correlation with assisted reproductive procedures.

\section{CONCLUSIONS}

$\mathrm{D}$ ata from the literature show that ART has an increased safety and efficacy for treating infertility and it also increases the chances of obtaining a pregnancy in this population. It has been shown that the implementation of single embryo transfer protocols had significantly contributed to reducing the risk of obstetric and perinatal complications compared to multiple pregnancies.

Simultaneously, it has been shown that certain reproductive techniques were associated with an increased risk of pregnancy complications. Thus, the transfer of a blastocyst stage embryo allows the selection of high-quality embryos, but it increases the incidence of prematurity and low birth weight in this group. Cryopreservation techniques reduce the risk of ovarian hyperstimulation syndrome and allow an effective synchronization between the embryo and endometrium, thus reducing the incidence of prematurity. However, it has been observed to increase the risk of hypertensive complications in pregnancy, large for gestational age, macrosomia and perinatal mortality. For this reason, it is necessary to limit the use of freeze-all protocols to specific groups of patients who can truly benefit from this technique. Regarding the use of ICSI, it has been shown that it increases the risk of congenital anomalies compared with IVF.

Studies have also shown that for certain complications in pregnancy, parental characteristics, the etiology of infertility and population of subfertility women could influence the prognosis. According to relevant studies, ART is not a direct causal factor of pregnancy and perinatal complications, but it is an additional risk factor for this population of women. This is why ART should become an important observational marker for clinicians, to include these pregnancies in risk categories and establish a standardized methodology for the follow-up of the mother and newborn.

Conflicts of interest: none declared. Financial support: none declared. 


\section{References}

1. Adamson GD, de Mouzon J, Chambers GM, et al. International Committee for Monitoring Assisted Reproductive Technology: world report on assisted reproductive technology, 2011. Fertil Steril 2018;110:1067-1080.

2. European IVF-monitoring Consortium (EIM) $\ddagger$ for the European Society of Human Reproduction and Embryology (ESHRE), Wyns C, Bergh C, Calhaz-Jorge C, De Geyter C, Kupka MS, Motrenko T, Rugescu I, Smeenk J, Tandler-Schneider A, Vidakovic S, \& Goossens V. (2020). ART in Europe, 2016: results generated from European registries by ESHRE. Human Reproduction Open 2020(3), hoaa032.

3. Wang AY, Safi N, Ali F, et al. Neonatal outcomes among twins following assisted reproductive technology: an Australian populationbased retrospective cohort study. BMC Pregnancy Childbirth 2018;18:320.

4. Lei LL, Lan YL, Wang SY, et al. Perinatal complications and live-birth outcomes following assisted reproductive technology: a retrospective cohort study. Chin Med J (Engl). 2019;132:2408-2416.

5. Roque $M$, Valle $M$, Sampaio $M$, et al. Obstetric outcomes after fresh versus frozen-thawed embryo transfers: A systematic review and meta-analysis. JBRA Assist Reprod 2018;22:253-260.

6. Henningsen AA, Gissler M, Skjaerven $\mathrm{R}$, et al. Trends in perinatal health after assisted reproduction: a Nordic study from the CoNARTaS group. Hum Reprod 2015;30:710-716.

7. Almasi-Hashiani A, Omani-Samani R, Mohammadi M, et al. Assisted reproductive technology and the risk of preeclampsia: an updated systematic review and meta-analysis. BMC Pregnancy Childbirth 2019;19:149.

8. Gui J, Ling $Z$, Hou $X$, et al. In vitro fertilization is associated with the onset and progression of preeclampsia. Placenta 2020;89:50-57.

9. Thomopoulos C, Tsioufis C, Michalopoulou H, et al. Assisted reproductive technology and pregnancy-related hypertensive complications: a systematic review. J Hum Hypertens 2013;27:148-157.

10. Maheshwari A, Pandey S, Amalraj Raja E, et al. Is frozen embryo transfer better for mothers and babies? Can cumulative meta-analysis provide a definitive answer?

Hum Reprod Update 2018;24:35-58.

11. Sha $T$, Yin $X$, Cheng $W$, et al.
Pregnancy-related complications and perinatal outcomes resulting from transfer of cryopreserved versus fresh embryos in vitro fertilization: a metaanalysis.

Fertil Steril 2018;109:330-342.e9.

12. Luke B, Brown MB, Eisenberg ML, et al. In vitro fertilization and risk for hypertensive disorders of pregnancy: associations with treatment parameters. Am J Obstet Gynecol 2020;222:350.e1-350.e13.

13. Moreno-Sepulveda J, Checa MA. Risk of adverse perinatal outcomes after oocyte donation: a systematic review and meta-analysis [published correction appears in J Assist Reprod Genet 2020;37:239] J Assist Reprod Genet 2019;36:2017-2037.

14. Berntsen S, Larsen EC, la Cour Freiesleben N, et al. Pregnancy outcomes following oocyte donation. Best Pract Res Clin Obstet Gynaecol. 2021;70:81-91.

15. Schwarze JE, Borda $P$, Vásquez $P$, et al. Is the risk of preeclampsia higher in donor oocyte pregnancies? A systematic review and meta-analysis. JBRA Assist Reprod 2018;22:15-19.

16. Zaat $T$, Zagers $M$, Mol F, et al. Fresh versus frozen embryo transfers in assisted reproduction. Cochrane Database Syst Rev 2021;2:CD011184.

17. Bosdou JK, Venetis CA, Tarlatzis BC, et al. Higher probability of live-birth in high, but not normal, responders after first frozen-embryo transfer in a freeze-only cycle strategy compared to fresh-embryo transfer: a meta-analysis. Hum Reprod 2019;34:491-505.

18. Roque M, Haahr T, Geber S, et al. Fresh versus elective frozen embryo transfer in IVF/ICSI cycles: a systematic review and meta-analysis of reproductive outcomes.

Hum Reprod Update 2019;25:2-14.

19. Singh B, Reschke L, Segars J, et al. Frozen-thawed embryo transfer: the potential importance of the corpus luteum in preventing obstetrical complications. Fertil Steril 2020;113:252-257.

20. von Versen-Höynck F, Schaub AM, Chi YY, et al. Increased Preeclampsia Risk and Reduced Aortic Compliance With In Vitro Fertilization Cycles in the Absence of a Corpus Luteum. Hypertension 2019;73:640-649.

21. Wang YA, Nikravan R, Smith HC et al. Higher prevalence of gestational diabetes mellitus following assisted reproduction technology treatment. Hum Reprod 2013;28:2554-2561.

22. Bosdou JK, Anagnostis P, Goulis DG, et al. Risk of gestational diabetes mellitus in women achieving singleton pregnancy spontaneously or after ART: a systematic review and meta-analysis. Hum Reprod Update 2020;26:514-544.

23. Sha $T$, Wang $X$, Cheng $W$, et al. A meta-analysis of pregnancy-related outcomes and complications in women with polycystic ovary syndrome undergoing IVF. Reprod Biomed Online 2019;39:281-293.

24. Vermey BG, Buchanan A, Chambers GM, et al. Are singleton pregnancies after assisted reproduction technology (ART) associated with a higher risk of placental anomalies compared with non-ART singleton pregnancies? A systematic review and meta-analysis. BJOG 2019;126:209-218.

25. Karami M, Jenabi E, Fereidooni B. The association of placenta previa and assisted reproductive techniques: a meta-analysis [published correction appears in

J Matern Fetal Neonatal Med 2019;32:4191]. J Matern Fetal Neonatal Med 2018;31:1940-1947.

26. Spangmose AL, Ginström Ernstad E, Malchau S, et al. Obstetric and perinatal risks in 4601 singletons and 884 twins conceived after fresh blastocyst transfers: a Nordic study from the CoNARTaS group. Hum Reprod 2020;35:805-815.

27. Dar S, Librach CL, Gunby J, et al. Increased risk of preterm birth in singleton pregnancies after blastocyst versus Day 3 embryo transfer: Canadian ART Register (CARTR) analysis. Hum Reprod 2013;28:924-928.

28. Madrazo-Cabo JM, León-Durán GA, Oliveros-Montiel A, et al. Complications in pregnancies achieved by assisted reproduction. Gac Med Mex 2020;156:156-163.

29. Pinborg A, Wennerholm UB, Romundstad LB, et al. Why do singletons conceived after assisted reproduction technology have adverse perinatal outcome? Systematic review and meta-analysis.

Hum Reprod Update 2013;19:87-104.

30. Cavoretto $P$, Candiani M, Giorgione V, et al. Risk of spontaneous preterm birth in singleton pregnancies conceived after IVF/ICSI treatment: meta-analysis of cohort studies.

Ultrasound Obstet Gynecol 2018;51:43-53. 


\section{Glujovsky D, Farquhar C,} Quinteiro Retamar AM, et al. Cleavage stage versus blastocyst stage embryo transfer in assisted reproductive technology.

The Cochrane Database of Systematic Reviews 2016: CD002118-CD002118.

32. Levi-Setti PE, Cirillo F, Smeraldi A, et al. No advantage of fresh blastocyst versus cleavage stage embryo transfer in women under the age of 39: a randomized controlled study. J Assist Reprod Genet 2018;35:457-465.

33. Aiken C, Brockelsby J. Fetal and maternal consequences of pregnancies conceived using ART.

Fetal and Maternal Medicine Review 2014;25:281-294.

34. Romundstad LB, Romundstad PR, Sunde A, et al. Effects of technology or maternal factors on perinatal outcome after assisted fertilisation: a populationbased cohort study.

Lancet 2008;372:737-743.

35. Reig A, Seli E. The association between assisted reproductive technologies and low birth weight.
Curr Opin Obstet Gynecol 2019;31:183-187.

36. Castillo CM, Johnstone ED, Horne G, et al. Associations of IVF singleton birthweight and gestation with clinical treatment and laboratory factors: a multicentre cohort study. Hum Reprod 2020;35:2860-2870.

37. Berntsen S, Pinborg A. Large for gestational age and macrosomia in singletons born after frozen/thawed embryo transfer (FET) in assisted reproductive technology (ART). Birth Defects Res 2018;110: 630-643.

38. Orvieto R, Kirshenbaum M, Gleicher N. Is Embryo Cryopreservation Causing Macrosomia-and What Else? Front Endocrinol (Lausanne) 2020;11:19.

39. Chen L, Yang T, Zheng Z, et al. Birth prevalence of congenital malformations in singleton pregnancies resulting from in vitro fertilization/intracytoplasmic sperm injection worldwide: a systematic review and meta-analysis. Arch Gynecol Obstet 2018;297:1115-1130.

40. Zheng Z, Chen L, Yang T, et al. Multiple pregnancies achieved with IVF/ICSI and risk of specific congenital malformations: a meta-analysis of cohort studies. Reprod Biomed Online 2018;36:472-482.

41. Zhao J, Yan Y, Huang X, et al. Do the children born after assisted reproductive technology have an increased risk of birth defects? A systematic review and meta-analysis. J Matern Fetal Neona 2020;33:322-333.

42. Giorgione V, Parazzini F, Fesslova V, et al. Congenital heart defects in IVF/ICSI pregnancy: systematic review and meta-analysis.

Ultrasound Obstet Gynecol 2018;51:33-42.

43. Davies MJ, Moore VM, Willson KJ, et al. Reproductive technologies and the risk of birth defects. N Engl J Med 2012;366:1803-1813.

44. Wennerholm UB, Bergh C. Perinatal outcome in children born after assisted reproductive technologies. Ups J Med Sci 2020;125:158-166.

45. Cortessis VK, Azadian M, Buxbaum J, et al. Comprehensive meta-analysis reveals association between multiple imprinting disorders and conception by assisted reproductive technology. J Assist Reprod Genet 2018;35:943-952. 